\title{
Nutritional issues in a diabetic patient with Kearns-Sayre syndrome
}

\author{
Alicja Szwilling', Katarzyna Dzygalo1(i) and Jędrzej Nowaczyk²(i) \\ 1Department of Pediatric Diabetology and Pediatrics, Children Teaching Clinical Hospitaland 2Department of \\ Pediatrics, Medical University of Warsaw, Warszawa, Poland
}

Correspondence should be addressed to J Nowaczyk Email

jnowaczyk@wum.edu.pl

\section{Summary}

Kearns-Sayre syndrome (KSS) is a multi-system mitochondrial disease with wide clinical presentation. We describe the case of a 16-year-old girl with KSS accompanied by insulin-dependent diabetes, eosinophilic esophagitis (EoE), Fanconi syndrome, insufficiency of parathyroid gland and severe nutritional problems. Based on recent knowledge, ketogenic diet was introduced to improve metabolic and neurological condition, however in our patient we observed its bad consequences. Unresolved nutritional disorders forced us to proceed with esophagogastroduodenoscopy which revealed EoE. PEG procedure was performed and elemental diet with PPI's was introduced leading to general improvement in the patient's health condition.

\section{Learning points:}

- Nutrition is an important factor in supportive care of patients with KSS.

- Ketogenic diet in patients affected by mitochondrial diseases and diabetes requires careful selection and monitoring.

- To the best of our knowledge, this is the first case that shows the coexistence of EoE, insulin-dependent diabetes and KSS.

\section{Background}

KSS is a rare multi-system mitochondrial disease. Three cardinal features of KSS involve onset before 20 years of age, progressive external ophthalmoplegia and bilateral pigmentary retinopathy and needs to be accompanied by at least one of below disorders like CNS disfunction (e.g. cerebellar ataxia), cognitive impairment, increased cerebrospinal fluid protein level, hearing loss, tremor, cardiomyopathy, short stature, endocrine dysfunction, lactic acidosis, emesis, aortic insufficiency, subaortic septum hypertrophy, right bundle-branch block, double vision or white matter lesions (1).

Some authors recommend using coenzyme Q10 and introducing specific dietary management in KSS treatment. Using a ketogenic diet is one of the suggested dietary propositions. Ketogenic diet stimulates mitochondrial biogenesis, improves mitochondrial function and decreases oxidative stress (2). This diet increases circulating level of ketone bodies. Ketogenic diet may be beneficial in conditions related to mitochondrial impairment, like diabetes, epilepsy and mitochondrial disorders (3).

\section{Case presentation}

A 9-year-old girl was admitted to our department with polyuria, polydipsia and weight loss of approximately $1 \mathrm{~kg}$. Laboratory tests did not reveal features of ketoacidosis. Insulin-dependent diabetes mellitus with negative immune markers was diagnosed. In addition, failure to thrive with BMI $13 \mathrm{~kg} / \mathrm{m}^{2}$ (Z-Score -2,47), ptosis, eye 
movement impairment, diplopia, decreased muscle tone, poorly expressed deep tendon reflexes and cognitive disorders were found. A few months before the diabetes onset, she started complaining of chronic headaches with visual disturbances, ocular palsy, photophobia and phonophobia without vomiting or nausea. Normal growth and psychomotor development were observed until the age of 3 . From the age of 4 , asymmetrical ptosis was revealed. In prospective observations, the patient presented symptoms of severe headaches, motor coordination disabilities, periodical vision disturbances and tremors of the head and limbs exacerbated over time. Seizure-like episodes were reported. At the age of 10, brain MRI showed abnormalities characteristic of spongiform group of mitochondrial diseases. An abnormal EEG sleep study was found and treatment with lamotrigine was introduced. Given the clinical and radiological findings supporting the suspicion of KSS, genetic testing was performed confirming the diagnosis. A blood mitochondrial DNA study identified deletion, which according to the literature is responsible for about $90 \%$ of cases of KSS.

At the age of 13, she was admitted in critical condition due to stroke-like episode. Observed symptoms were: sudden confusion, severe headache and weakness in left arm and the face on left side and abnormal breathing pattern. Laboratory tests showed elevated lactate level and electrolyte disturbances. A few months later, she had lost consciousness with associated hypocalcemia. Diagnosis of primary hypoparathyroidism was established and treatment with calcium supplementation was introduced. At the age of 14.5, patient developed Fanconi syndrome with nephrocalcinosis and retinal dystrophy with progressive vision impairment was diagnosed. Figure 1 presents photos our patient at the age of 13 .

Diabetes control remained inadequate during the entire observation period (mean HbA1c about 7.5\%) with onset $\mathrm{HbA} 1 \mathrm{c}$ of $11.2 \%$ (Figs 2 and 3a). The patient was treated with continuous subcutaneous insulin infusion from diagnosis. Mean insulin requirement was $0.63 \mathrm{U} / \mathrm{kg} /$ day. Patient developed obsessive-compulsive and anxiety disorders with selective eating disorder. She ate mainly dairy processed meals such as sweet homogenized vanilla cheese and omitted whole meals. A high-caloric formula $150 \mathrm{~mL}$ five times/day was added to her normal diet due to failure to thrive. The girl reported severe pain in legs during hypoglycemic episodes. All these factors contribute to worsening of diabetes metabolic control.

At the age of 16 after consultation with the hospice physician, parents decided to introduce classic ketogenic

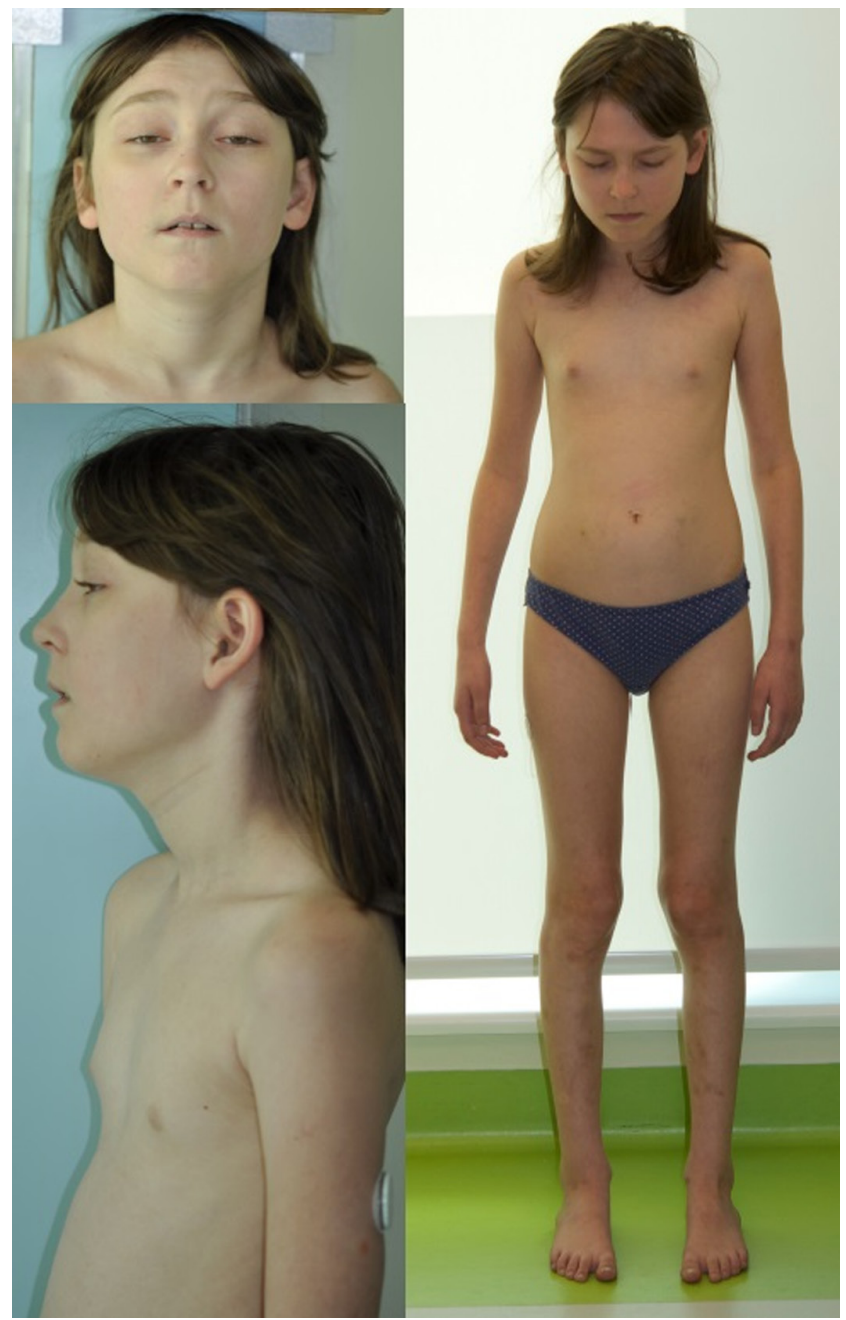

\section{Figure 1}

Actual pictures of patient at the age of 13 years

diet to our patient. It was decided to initiate a dietary regimen using non-fasting protocol. Ketosis was induced by increasing ketogenic ratio, however, the patient was unable to achieve the 4:1 target ratio. Introduction of the diet was eventually interrupted at the stage of $2: 1$ ratio. Detailed composition of the ketogenic diet is shown in Table 1. Moreover the patient's parents were informed to measure ketone bodies levels in urine several times a week, however, they didn't follow those recommendations. Urine ketones measurement was chosen for economic reasons (4). The purpose of ketogenic diet was to improve neurological condition and glycemic control. In the one week of $1900 \mathrm{kcal}$ ketogenic diet, insulin requirement decreased to minimal doses (from 0.65 to $0.27 \mathrm{IU} / \mathrm{kg} /$ day) (Fig. 4) and the patient achieved good metabolic control with stable glycemia and weight gain $(+0.4 \mathrm{~kg})$ (Fig. 3b). Tremors and 


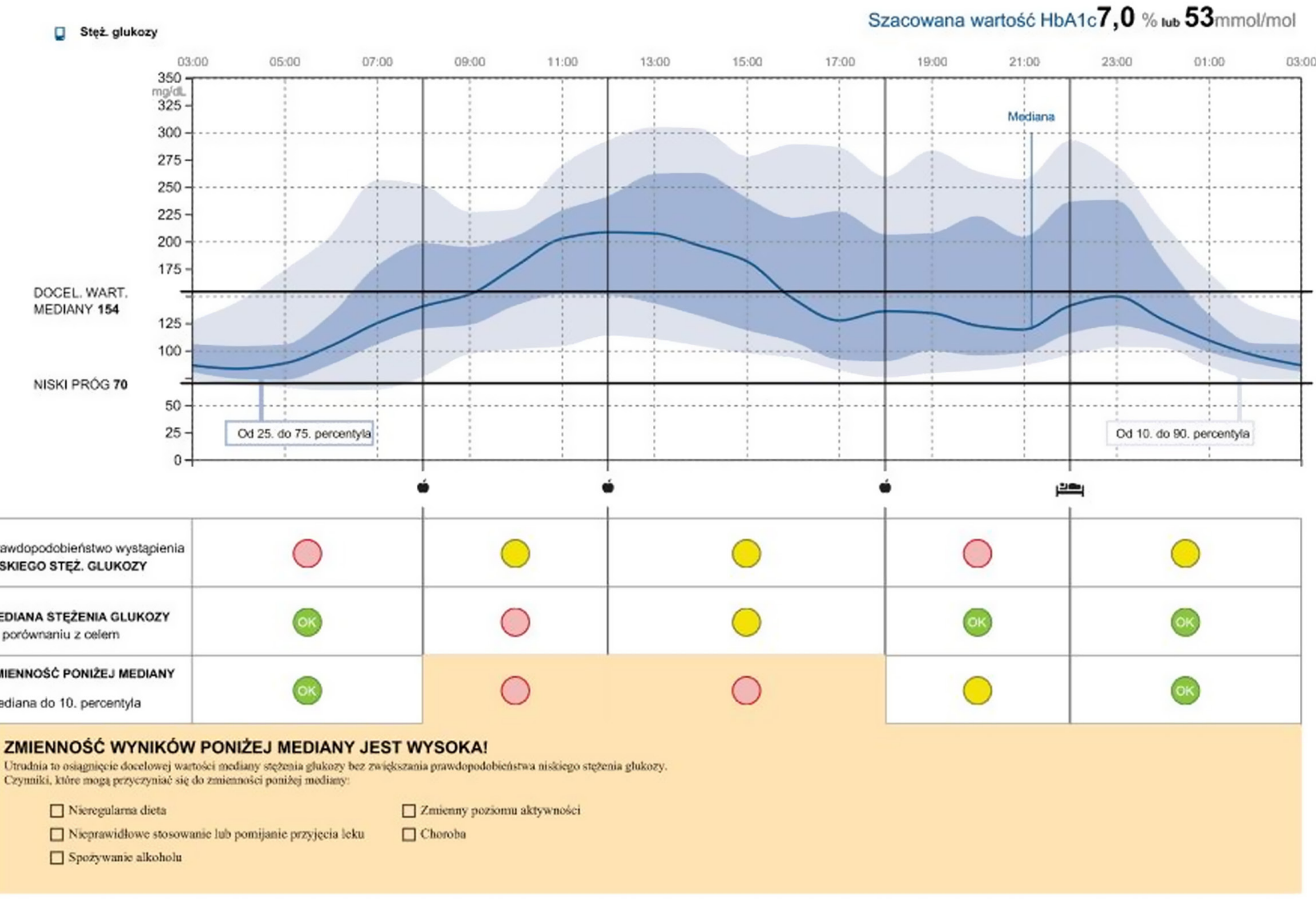

Figure 2

Glycemic pattern from flash glucose monitoring system - original polish version.

absence seizures resolved, nevertheless the girl's general condition worsened. She became apathetic, drowsy and disorientated. As ketogenic diet brought more losses than benefits in this case, previous diet was reinstituted immediately. Glycemic control had worsened, with mean glycemia about $180 \mathrm{mg} / \mathrm{dL}$, however, the patient's general health instantly improved.

As mentioned before, our patient has presented a reluctance to eat from early childhood but the feeding problems were exacerbated from the age of 14 . At the age of 16.5 she was admitted to our department due to dysphagia, weight loss ( $2 \mathrm{~kg}$ in 3 months) and abdominal pain presented a few days prior to the admission. Her current BMI was $14.9 \mathrm{~kg} / \mathrm{m}^{2}$ (Z-Score -3.34). Presented symptoms were suspected to be related with oral and pharyngeal disabilities as a progression of the main underlying disease. The esophagogastroduodenoscopy (EGD) preceded the procedure of percutaneous endoscopic gastrostomy (PEG) placing. Based on biopsy, eosinophilic esophagitis (EoE) was confirmed. The proton pump inhibitor therapy and high-energy elemental formula feeding was initiated. After a few days of progressive health state improvement, the patient started gaining weight gradually. Her parents decided on their own to increase the serving size of the elemental formula. After 2 weeks, the girl started complaining of severe abdominal pain connected with feeding. On admission to our department she suffered from pain in the upper abdomen, intensifying on palpation. No changes in ultrasound exam of the abdomein was shown. Lipase level was elevated to 427 U/L. Reduction of feeding serving size was recommended by gastroenterologist leading immediately to health improvement and stabilization of glycemia, although normalization of pancreatic enzymes was not achieved.

Finally, she gained weight ( $+5 \mathrm{~kg}$ in 6 months) with actual BMI of $17.1 \mathrm{~kg} / \mathrm{m}^{2}$ (Z-Score -2.47).

\section{Investigation}

A blood mitochondrial DNA study detected heteroplasmy rate lower than $<30 \%$ and reduced by $40-50 \%$ level of ligation probes located in the area m.7120_m.14068 including genes MTCOX1, MTCOX2, MTATP6, MTCOX3, MTND3, MTND4, iMTND5 confirming the diagnosis. 
A

CZW 14 lis

घ Stęż. glukozy mołal

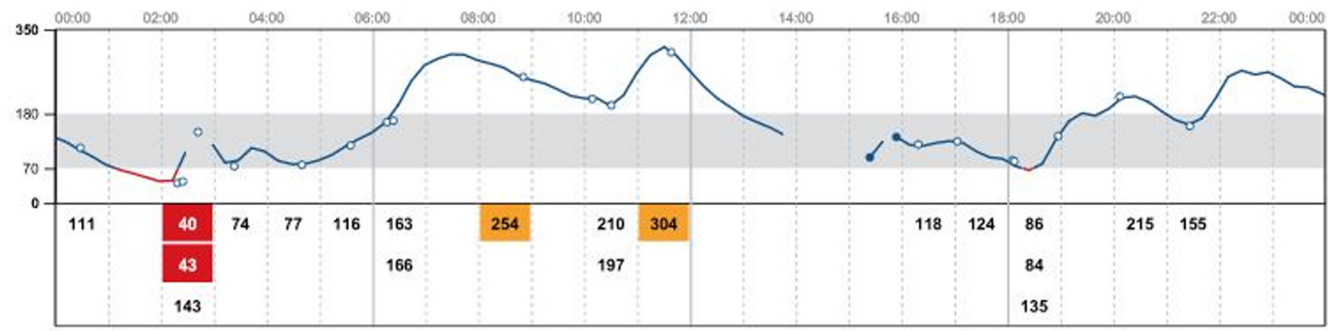

PT 15 lis

¿ Stęż. glukozy mơla

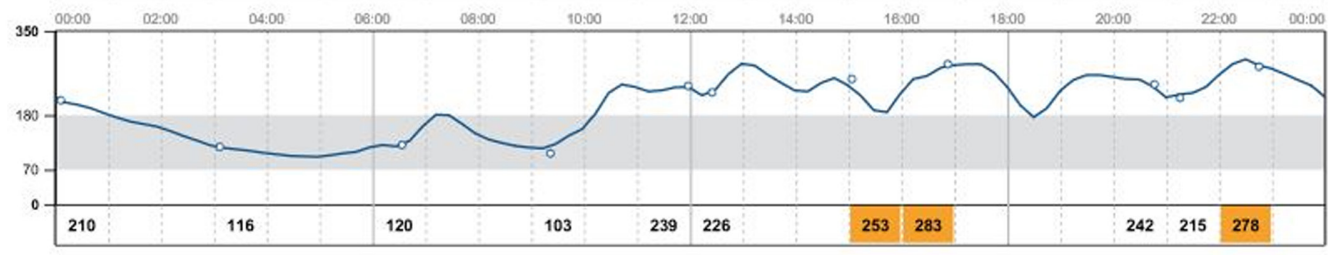

SOB 16 lis

D Stęz. glukozy mgldL

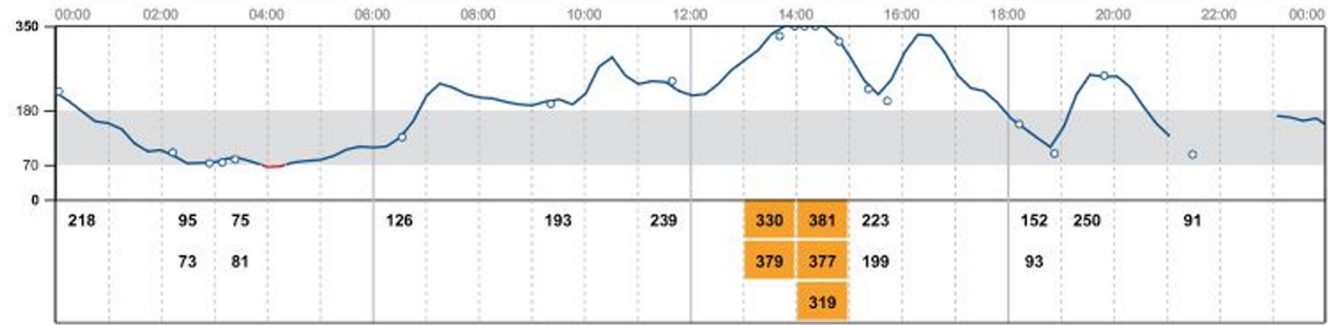

$\mathrm{B}$

SR 20 lis

Đ Stęż. glukozy mọdL

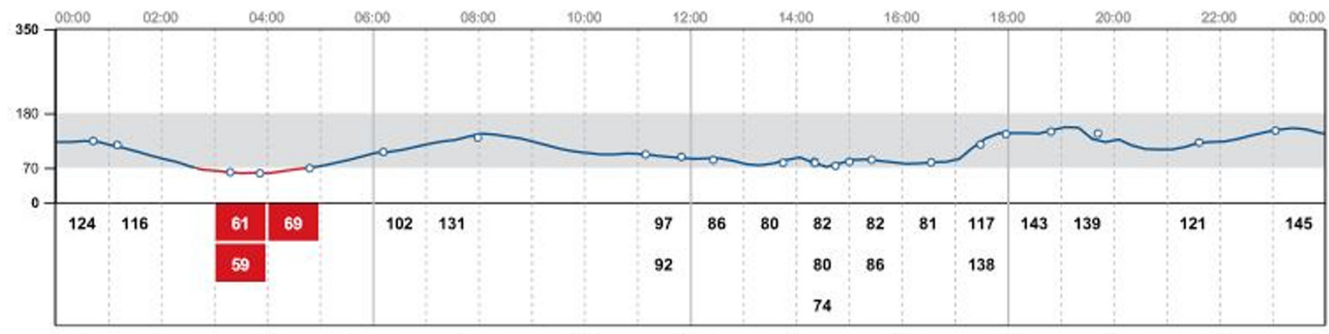

CZW 21 lis

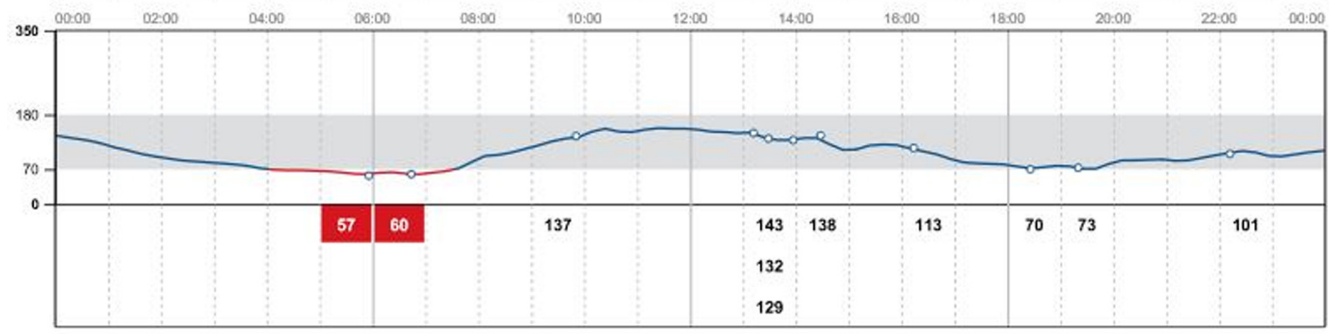

PT 22 lis

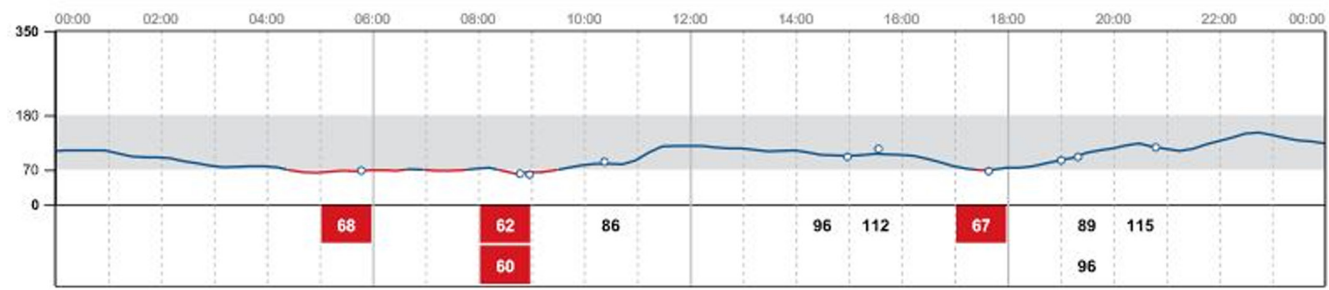

Figure 3

(A) Recordings of patient's glycemias from flash glucose monitoring system before introduction of ketogenic diet - original polish version. (B) Recordings of patient's glycemias from flash glucose monitoring system after introduction of ketogenic diet - original polish version. 
Table 1 Detailed composition of the ketogenic diet.

\begin{tabular}{lr}
\hline Energy (kcal) & 1900 \\
Fat (g) & 173 \\
Protein (g) & 74 \\
Carbohydrates $(\mathrm{g})$ & 16 \\
$\mathrm{Na}(\mathrm{mg})$ & 2500 \\
$\mathrm{~K}(\mathrm{mg})$ & 4700 \\
$\mathrm{Mg}(\mathrm{mg})$ & 1040 \\
$\mathrm{Ca}(\mathrm{mg})$ & 2000 \\
\hline
\end{tabular}

The visual findings in EGD include linear furrows, narrows in upper and middle part of esophagus and white exudates. There were no abnormal findings from the stomach to duodenum. Helicobacter pylori infection was excluded by negative rapid urease test. Esophageal mucosal biopsy specimens contained 80-100 intraepithelial eosinophils per high-power microscopic field, which led to EoE diagnosis.

\section{Treatment}

Long-term biochemical monitoring of electrolytes levels was performed because of endocrinological and nephrological disorders. Long-term follow-up in the diabetology clinic regarding IDDM was also done.

\section{Outcome and follow-up}

Due to the progressive nature of her disease, the patinet needs to be under the care of a multidisciplinary team. Recently she did not present any episodes of acute complications, but her neurological condition was worsening.

\section{Discussion}

KSS is a rare disease. There are only few descriptions of cases of KSS patients with insulin-dependent diabetes (IDDM). IDDM in patients with KSS syndrome may be caused by several factors including decreased insulin secretion, increased insulin resistance, abnormal development of pancreatic islet cell architecture, autoimmune pancreas destruction and secondary diabetes due to recurrent pancreatitis (5). Poulton et al. found postmortem no beta cells in pancreas of a patient with KSS who died at the age

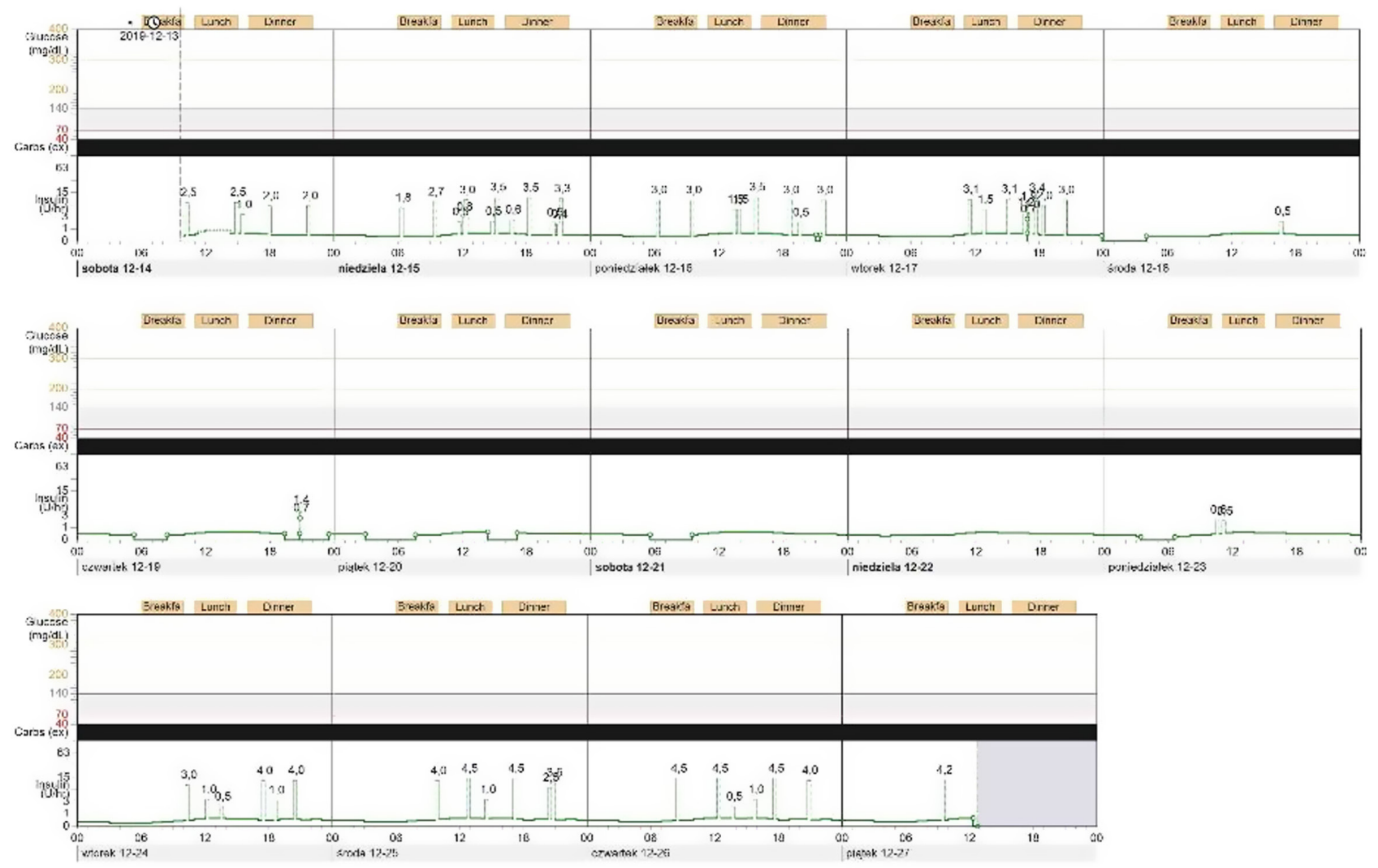

Figure 4

Recordings of patient's personal insulin pump - period before and after introduction of ketogenic diet. 
of 20 years (6). Ho et al. described two children with KSS and IDDM onset at the age of 4 and 11 years (5). Both children were fed via gastrostomy. They were treated with basal-bolus therapy with insulin requirement between 0.5 and 0.8 units $/ \mathrm{kg} /$ day and HbA1c range between 7.9 and $9.4 \%$. Our patient used CSII with insulin requirement of 0.65 units $/ \mathrm{kg} /$ day and poor metabolic control (HbA1c 7.8\%).

Although our patient has been using modern technologies (CSII and FGM), she has not achieved the recommended diabetes control. According to the ISPAD guidelines, the therapeutic target of $\mathrm{HbA1c}$ of $7.0 \%$ is recommended (7). Due to selective eating disorder and omitting whole meals, it was difficult to establish proper prandial insulin dose for the patient. The girl mainly ate dairy processed meals such as sweet homogenized vanilla cheese which caused significant glucose fluctuations. Wide glucose fluctuations and chronic hyperglycemia and hypoglycemia have adverse effects on neurocognitive function and brain structure in children with diabetes.

Nutritional management in children with diabetes can improve metabolic outcomes. Ketogenic diet can reduce hyperglycemia and improve HbA1c in children with T1D, but most children discontinued this diet as intolerable (8). Parents reported different side effects such as impaired growth, delayed bone age, low energy, low mood, poor sleep, amenorrhea, hypoglycemia and elevated fasting cholesterol.

Some children with T1D are treated with ketogenic diet due to seizure disorders. Aylward et al. described a 4 -year-old child with type 1 diabetes and myoclonic astatic epilepsy who was treated successfully with a ketogenic diet for 6 years (9). The patient was fed via gastrostomy which facilitated adherence to ketogenic diet. Before the introduciton of the ketogenic diet, seizures continued despite therapeutic levels of five antiseizure medications. Since initiation of the diet, the patient did not have any drop attacks or myoclonic astatic seizures and continued to have the occasional brief nocturnal seizure. Her cognitive function significantly improved. She achieved stable metabolic control with HbA1c between 5.7-6.4\% with no episodes of severe hypoglycemia or diabetic ketoacidosis. The promising results of ketogenic diet in the treatment of children with epilepsy and mitochondrial disorders has been confirmed in the literature (10). However, children with diabetes using ketogenic diet should be under special control. Restricted carbohydrate diets are not recommended by ISPAD in children with T1D as it may result in growth failure, higher cardiovascular risk metabolic profile and increased risk of hypoglycemia. in diabetic children on ketogenic diet, insulin requirement decreases, and low insulin promotes fatty acid oxidation and ketosis. Therefore, it is necessary to differentiate between starvation-induced ketones and those increasing from insulin deficiency to avoid diabetic ketoacidosis. The ketogenic diet in our patient improved diabetes control.

EoE is a rare disease which has so far unknown comorbidity in KSS patients. Recommended treatment based on the American College of Allergy, Asthma and Immunology is elemental diet, proton pump inhibitors and glucocorticosteroids. In order to avoid the potential impact of glucocorticosteroids on glycemic control, we decided to initiate therapy with diet and PPI's with good therapeutic effect.

\section{Conclusions}

Nutrition is an important factor in supportive care of patients with KSS. Besides of known reports describing beneficial effects of ketogenic diet in mitochondrial diseases, in our patient we revealed its bad consequences. It is crucial to be aware of the necessity of careful selection and monitoring of ketogenic diet in patients affected by mitochondrial diseases.

Moreover, our case emphasizes the need to consider early enteral tube feeding implantation in patients with progressive failure to thrive and additional nutritional problems as early as possible to improve their quality of life, diminish cumbersome symptoms from gastrointestinal tract, ameliorate growth failure and improve the patient's general condition.

Coexistence of diabetes with KSS may implicate additional difficulties in the therapy management.

KSS is a complex disease requiring multidisciplinary care of a patient. Modifying the treatable effects may ease the disease burden and improve the patient and parents' quality of life.

\section{Declaration of interest}

The authors declare that there is no conflict of interest that could be perceived as prejudicing the impartiality of this case report.

\section{Funding}

This work did not receive any specific grant from any funding agency in the public, commercial, or not-for-profit sector.

\section{Patient consent}

Written informed consent has been obtained from the patient. 


\section{Authors contribution statement}

All authors included in this article made substantial contributions to the analysis of the data included, as well as assisted with critical revisions of the writing, and approved the final version for submission for publication.

\section{References}

1 Khambatta S, Nguyen DL, Beckman TJ \& Wittich CM. Kearns-Sayre syndrome: a case series of 35 adults and children. International Journal of General Medicine 20147 325-332. (https://doi.org/10.2147/IJGM. S65560)

2 Branco AF, Ferreira A, Simões RF, Magalhães-Novais S, Zehowski C, Cope E, Silva AM, Pereira D, Sardão VA \& Cunha-Oliveira T.

Ketogenic diets: from cancer to mitochondrial diseases and beyond. European Journal of Clinical Investigation 201646 285-298. (https:// doi.org/10.1111/eci.12591)

3 Ahola S, Auranen M, Isohanni P, Niemisalo S, Urho N, Buzkova J, Velagapudi V, Lundbom N, Hakkarainen A, Muurinen T, et al. Modified Atkins diet induces subacute selective ragged-red-fiber lysis in mitochondrial myopathy patients. EMBO Molecular Medicine 2016 8 1234-1247. (https://doi.org/10.15252/emmm.201606592)

4 Kossoff EH, Zupec-Kania BA, Auvin S, Ballaban-Gil KR, Christina Bergqvist AG, Blackford R, Buchhalter JR, Caraballo RH, Cross JH, Dahlin MG, et al. Charlie foundation; Matthew's Friends; Practice
Committee of the Child Neurology Society. Epilepsia Open 20183 175-192. (https://doi.org/10.1002/epi4.12225)

5 Ho J, Pacaud D, Rakic M \& Khan A. Diabetes in pediatric patients with Kearns-Sayre syndrome: clinical presentation of 2 cases and a review of pathophysiology. Canadian Journal of Diabetes 201438 225-228. (https://doi.org/10.1016/j.jcjd.2014.04.003)

6 Poulton J, O'Rahilly S, Morten KJ \& Clark A. Mitochondrial DNA, diabetes and pancreatic pathology in Kearns-Sayre syndrome. Diabetologia 199538 868-871. (https://doi.org/10.1007/ s001250050366)

7 DiMeglio LA, Acerini CL, Codner E, Craig ME, Hofer SE, Pillay K \& Maahs DM. ISPAD Clinical Practice Consensus Guidelines 2018: glycemic control targets and glucose monitoring for children, adolescents, and young adults with diabetes. Pediatric Diabetes 2018 19 105-114. (https://doi.org/10.1111/pedi.12737)

8 McClean AM, Montorio L, McLaughlin D, McGovern S \& Flanagan N. Can a ketogenic diet be safely used to improve glycaemic control in a child with type 1 diabetes? Archives of Disease in Childhood 2019104 501-504. (https://doi.org/10.1136/ archdischild-2018-314973)

9 Aylward NM, Shah N \& Sellers EA. The ketogenic diet for the treatment of myoclonic astatic epilepsy in a child with type 1 diabetes mellitus. Canadian Journal of Diabetes 201438 223-224. (https://doi.org/10.1016/j.jcjd.2014.05.002)

10 Paleologou E, Ismayilova N \& Kinali M. Use of the ketogenic diet to treat intractable epilepsy in mitochondrial disorders. Journal of Clinical Medicine 20176 56. (https://doi.org/10.3390/jcm6060056)

Received in final form 30 September 2020

Accepted 8 October 2020 